\title{
CURRENT WAYS TO HARVEST ENERGY USING A COMPUTER MOUSE
}

\author{
Frantisek Horvat ${ }^{a, *}, \operatorname{Michal~Cekan~}^{a}, \operatorname{Lukas~Soltes}^{a}, \operatorname{Peter~Biath~}^{a}$, \\ Branislav HUCKO ${ }^{a}$, IGOR JedinAK ${ }^{b}$ \\ a Slovak University of Technology, Namestie Slobody 17, Bratislava \\ ${ }^{b}$ Igor Jedinak, Sturova 1305/36, Kysucke Nove Mesto \\ * corresponding author: frantisek.horvat@stuba.sk
}

\begin{abstract}
This paper deals with the idea of an energy harvesting (EH) system that uses the mechanical energy from finger presses on the buttons of a computer mouse by means of a piezomaterial (PVF2). The piezomaterial is placed in the mouse at the interface between the button and the body. This paper reviews the parameters of the PVF2 piezomaterial and tests their possible implementation into EH systems utilizing these types of mechanical interactions. The paper tests the viability of two EH concepts: a battery management system, and a semi-autonomous system. A statistical estimate of the button operations is performed for various computer activities, showing that an average of up to 3300 mouse clicks per hour was produced for gaming applications, representing a tip frequency of $0.91 \mathrm{~Hz}$ on the PVF2 member. This frequency is tested on the PVF2 system, and an assessment of the two EH systems is reviewed. The results show that fully autonomous systems are not suitable for capturing low-frequency mechanical interactions, due to the parameters of current piezomaterials, and the resulting very long startup phase. However, a hybrid EH system which uses available power to initiate the circuit and eliminate the startup phase may be explored for future studies.
\end{abstract}

KEYwoRDS: Energy harvesting, computer mouse, piezomaterial, battery management.

\section{INTRODUCTION}

Many studies involving energy harvesting (EH) from human activity have concentrated on mounting an EH device to the body itself and using human motion to generate electrical energy [1]. However, there are other possible ways to obtain energy from human activity. Whether for work or for play, humans interact extensively with their personal computer (PC), and this interaction can be considered for EH. Interfacing with a PC typically involves a mouse and a keyboard, and depending on the activity, a computer mouse offers unique possibilities for $\mathrm{EH}$, particularly in the mechanisms that are involved. Studies have already been made for the potential application of $\mathrm{EH}$ circuits based on a piezomaterial, and have shown the possibility of applying it for battery management of cardiostimulators [2]. Similarly, the buttons on a computer mouse can be set up to introduce ideal displacements on a given piezomaterial for optimal energy generation. Considering the enormous number of people who interact with a PC every day, for work and for entertainment, on a large scale, the energy that is generated offers potential for $\mathrm{EH}[3]$.

\section{STUDY}

A piezomaterial can generate electricity, but the quantity of energy produced is a function of the frequency and the tip mass of the displaced material. To determine, on average, how many interactions humans perform with a computer mouse, a statistical analysis was carried out for various activities performed by 10 volunteers. Using a computer program that monitors mouse clicks per hour [4, each volunteer was asked to measure their interactions over one hour of typical work. Following this analysis, the same volunteers were asked to perform a measurement after one hour of gaming. The results are shown in Figure 1.

The table shows that, on an average, administrative work produces 170 mouse clicks per hour, which is essentially useless for EH. However, gaming produces a very much greater number of interactions - an average of 3300 clicks per hour. The values are highly dependent on the type of work being performed or on the game being played. It is obvious that gaming offers the greatest potential for generating energy. A suitable EH model, material, and configuration must also be considered.

\section{Method}

The most widely-used piezomaterial for $\mathrm{EH}$ is based on lead zirconate titanate $\left(\mathrm{Pb}\left(\mathrm{Zr}_{x} \mathrm{Ti}_{1-x}\right) \mathrm{O}_{3}\right.$ or $\mathrm{PZT}$. These structures offer very good performance, and they were therefore used in this study [5]. The cantilever loaded by the Dirac function in Figure 2 shows how the piezomaterial functions.

The PVF2 piezomaterial is placed at the root of this beam. A load and frequency are exciting this beam. The load at the end of the beam displaces the internal crystal structure, and this generates a charge. Higher tip masses are used to fine tune the region 


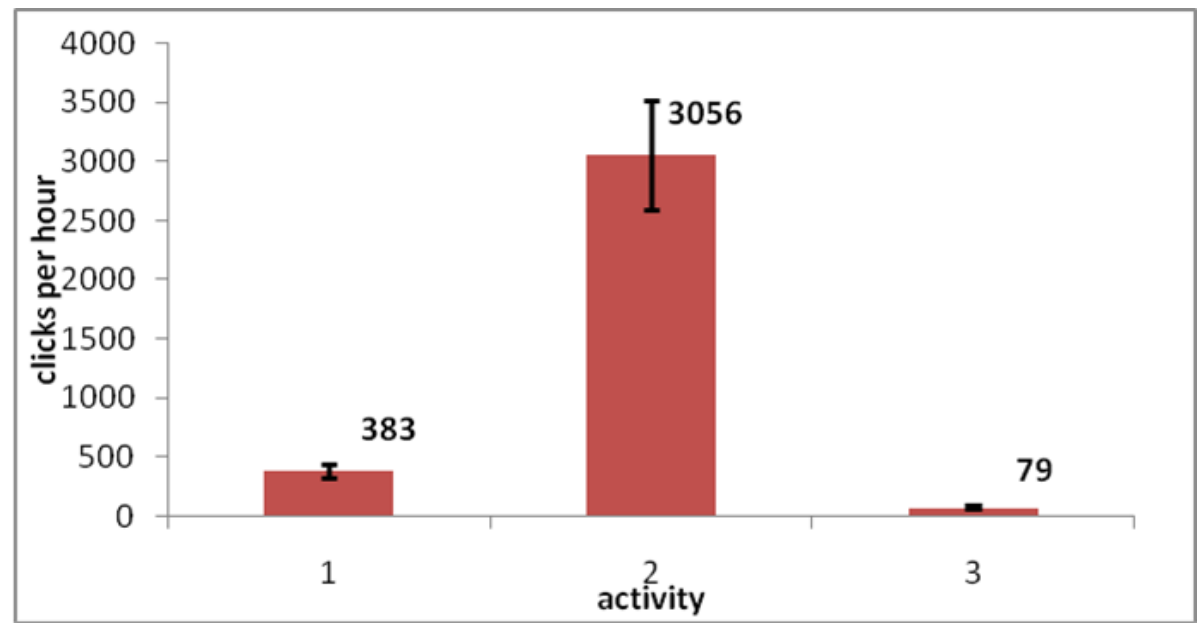

FIGURE 1. Statistical analysis of mouse clicks for various computer activities: 1 - administration, 2 - gaming, 3 programming.

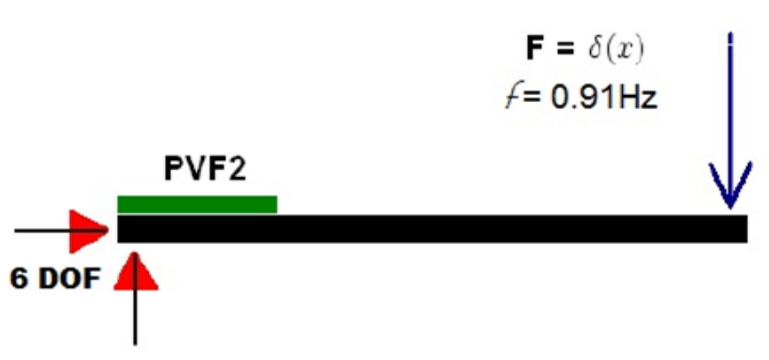

Figure 2. Cantilever beam loaded by a Dirac function.

of resonance in which the piezomaterial operates [5]. The corresponding voltage that is generated is used as an input into the EH circuit.

There are two EH methods, shown in Figure 3 , that can be implemented into the mouse circuit. Model 1 shows a system used for battery management (battery recharging or a charge extender), and model 2 shows the direct use of the generated energy in a semi-autonomous system.

In the analysis, piezomaterial with dimensions of $13 \times 25 \times 0.3 \mathrm{~mm}$ were considered. The PVF2 material that was used indicated higher impulse voltages at an optimal tip deflection of $\pm 1.5 \mathrm{~mm}$ and a higher tip mass. The piezomaterial is optimized for $30 \mathrm{~Hz}$ resonance. Higher tip mass reduced the required frequency to induce energy. The EH circuit from Linear Technologies was used, and the calculations were based on this circuit [6].

\section{Measurement And Results}

The PVF2 piezo-member was glued to the body of the mouse so that the tip of the PVF2 beam was positioned at the interface between the mouse button and body, as shown in Figure 4. Care was taken to ensure consistent tip deflection coinciding with the optimal deflection of the piezomaterial. The EH circuit was constructed according to LT specifications, with an output set at $1.8 \mathrm{~V}$. This circuit was placed

\section{Model 1:}

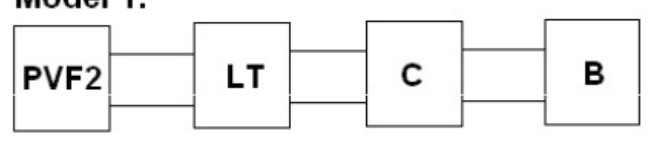

Model 2:

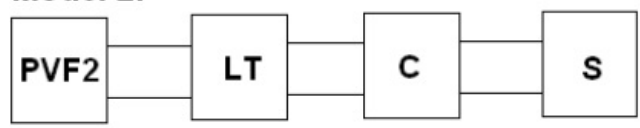

Figure 3. Possible energy harvesting systems, PVF2 - piezomaterial, LT - circuit required for $\mathrm{EH}, \mathrm{C}$ - capacitor, B - battery, $\mathrm{S}$ - input to mouse system.

in an open cavity of the mouse body, as is also shown in Figure 4

The mouse was then assembled, and the left button of the mouse was actuated to determine whether the $\mathrm{EH}$ system was functioning. Measurements were taken at the input and output of the LT-based EH circuit. On an average, the piezomaterial generated $320 \mathrm{mV}$ of input voltage per click, which is consistent with an approximately $30 \%$ reduction in expected values. At $0.91 \mathrm{~Hz}$ (gaming activities), the circuit start-up profile required approximately 2850 mouse clicks to accumulate enough energy to open the LT circuit. After this point, energy is passed through the circuit, and an output of $1.77 \mathrm{~V}$ is generated.

\section{Conclusions}

It must be stated that the frequency of mouse clicks is highly dependent on the type of activity. Some games require more use of the mouse than others. The same is true for certain working activities. The piezo-member was designed to operate at $30 \mathrm{~Hz}$. Because of this, the results show that the start-up process is too long, even when considering gaming activities (approximately 51 minutes at 0.91 clicks per second). Fully autonomous systems are therefore currently not possible, due to the long start-up profile and the in- 

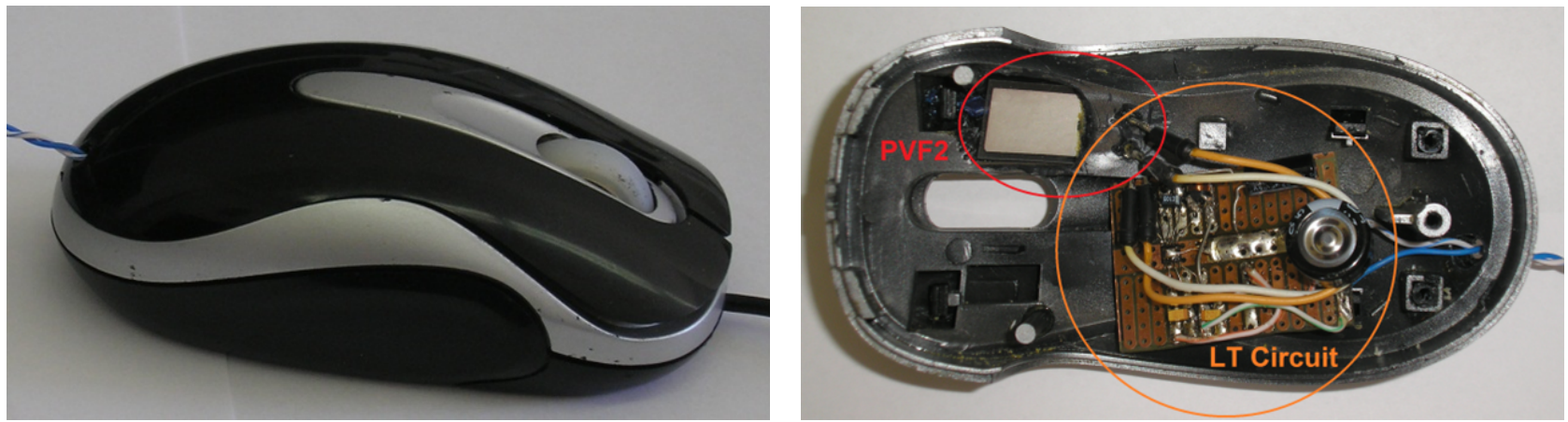

FiguRE 4. Implementation of the PVF2 piezomaterial on the left mouse button and the LT EH circuit.

herently high voltage dropout as charge is built. It should be stated that once the EH circuit overcomes the regulator start-up profile, it begins to release energy to the circuit output. Therefore, any further study could involve an EH mouse circuit operating as a hybrid system, where the regulator start-up profile is initiated by the available on-board power, and thus any clicking directly adds energy to the output of the circuit. However the character of the voltage drop suggests that this kind of system is not possible with the given piezomaterial and with low frequency input. It should be stated that the measurements were carried out on the left mouse button alone. A series of piezo-members mounted on both mouse buttons, or combined integration into other peripherals, e.g. the keyboard, could possibly sustain the charge in the system. Finally, current piezomaterial parameters are not suitable for such low frequency operations, and it is difficult to induce the required $30 \mathrm{~Hz}$ resonance on these members in these operations, due to the inconsistency of the impulses.

\section{REFERENCES}

[1] Riemer, R., Shapiro, A.: Biomechanical energy harvesting from human motion: theory, state of the art, design guidelines, and future directions, Journal of Neuroengineering and Rehabilitation, 2011, 8:22

[2] Karami, A., Inman, D.: Powering pacemakers from heartbeat vibrations using linear and nonlinear energy harvesters, American Institute of Physics, 2012, Lett. 100, 042901

[3] Horvat, F., Cekan, M., Soltes, L., Hucko, B.: Myska Prostriedok alebo zdroj?, In abstracts, Preveda, 2013, ISBN:78-80-970712-4-0.

[4] Codeplex, Application usage statistics, Project Hosting for Open Source Software. www. usagestats.codeplex.com

[5] Setter, N.: Piezoelectric materials in devices, EPFL Swiss Federal Institute of Technology, 2002, pp. 6-26.

[6] Linear Technology.: Piezoelectric energy harvesting power supply, data sheet, online: www.linear.com [2014-01-27]. 